\title{
Homoclinic Renormalization Group Flows, or When Relevant Operators Become Irrelevant
}

\author{
Christian B. Jepsen $\oplus^{1}$ and Fedor K. Popov $\circledast^{2}$ \\ ${ }^{1}$ Simons Center for Geometry and Physics, Stony Brook University, Stony Brook, New York 11794, USA \\ ${ }^{2}$ Department of Physics, Princeton University, Princeton, New Jersey 08544, USA
}

(Received 25 May 2021; accepted 23 August 2021; published 29 September 2021)

\begin{abstract}
We study an $\mathcal{N}=1$ supersymmetric quantum field theory with $O(M) \times O(N)$ symmetry. Working in $3-\epsilon$ dimensions, we calculate the beta functions up to second loop order and analyze in detail the renormalization group (RG) flow and its fixed points. We allow $N$ and $M$ to assume general real values, which results in them functioning as bifurcation parameters. In studying the behavior of the model in the space of $M$ and $N$, we demarcate the region where the RG flow is nonmonotonic and determine curves along which Hopf bifurcations take place. At a number of points in the space of $M$ and $N$ we find that the model exhibits an interesting phenomenon: at these points the RG flow possesses a fixed point located at real values of the coupling constants $g_{i}$ but with a stability matrix $\left(\partial \beta_{i} / \partial g_{j}\right)$ that is not diagonalizable and has a Jordan block of size two with zero eigenvalue. Such points correspond to logarithmic conformal field theories and represent Bogdanov-Takens bifurcations, a type of bifurcation known to give rise to a nearby homoclinic orbit - an RG flow that originates and terminates at the same fixed point. In the present example, we are able to employ analytic and numeric evidence to display the existence of the homoclinic RG flow.
\end{abstract}

DOI: $10.1103 /$ PhysRevLett.127.141602

Introduction.-Since the classic review by Kogut and Wilson [1] on the $\epsilon$ expansion and renormalization group (RG) flow, the general properties of RG flows have been the subject of active research. In the cases usually considered, once a theory starts flowing, it ends up at a fixed point where it is described by some conformal field theory (CFT). From a general point of view, the equations describing instances of RG flow form systems of autonomous differential equations, and the properties of such systems and flows they admit are well understood [2-5]. In particular, dynamical systems can exhibit flows more peculiar than that between distinct fixed points, and Kogut and Wilson speculated on the possibility of limit cycles, ergodic, and turbulent behavior in RG flow. Since then a number of monotonicity theorems have been proven that severely restrict the RG flow of unitary quantum field theories (QFTs). The first such theorem was Zamolodchikov's $c$ theorem [6], which establishes a function that interpolates between central charges at $2 d$ CFTs and decreases monotonically along RG flow. Analogous theorems were proven in four dimensions ( $a$ theorem) $[7,8]$ and three dimensions ( $F$ theorem) [9-11].

Published by the American Physical Society under the terms of the Creative Commons Attribution 4.0 International license. Further distribution of this work must maintain attribution to the author(s) and the published article's title, journal citation, and DOI. Funded by SCOAP ${ }^{3}$.
The monotonicity implied by these theorems excludes the possibility of limit cycles, except for a loophole pointed out in $[12,13]$ : multivalued $c$ functions. This loophole was used in deformed Wess-Zumino-Witten models [14-16], although the coupling constants pass between infinity and minus infinity in order to realize cyclic RG flow. There are also examples of cyclics RG flow in quantum mechanics [17-23].

Recently, Ref. [24] put forward a QFT of interacting symmetric traceless matrices transforming under the action of the $O(N)$ group, while allowing $N$ to assume noninteger values. $O(N)$ models for noninteger $N$, an idea widely used in polymer physics [25], had been previously given a formal definition in [26], which demonstrated the nonunitarity of these models. Hence, the $c, a, F$ theorems are no longer valid and do not constrain the RG flow, and consequently Ref. [24] was able to show that the considered model possesses a limit cycle for $N>N_{*} \approx 4.475$. The main tool used to make this discovery was Hopf's theorem [27], which guarantees the existence of a limit cycle in the vicinity of the codimension-one AndronovHopf bifurcation.

Turning to dynamical systems parametrized by two real numbers, codimension-two bifurcations can be used to prove the occurrence of yet other kinds of flow. Specifically, Bogdanov [28] and Takens [29] have established theorems by which, from properties of autonomous differential equations known only to second order in the 
dynamical variables, one can deduce the existence of homoclinic orbits, i.e., flow curves that connect a fixed point to itself. In addition to mild genericity conditions, the conditions for this bifurcation could be checked by studying the stability of fixed points, despite the fact that homoclinic orbits signal global bifurcations [2] since they arise when a limit cycle collides with a saddle point.

An interesting fact about homoclinic orbits is that they can be used to diagnose chaos. In applications of the theory of dynamical systems to physics, chaotic behavior [30] occurs in many instances, such as in turbulence [31,32], meteorology [33], and even in scattering amplitudes in string theory [34]. Usually, chaotic behavior is proven via numerical simulations. One of the few analytical tools that can hint at the emergence of chaos is a theorem due to Shilnikov [35] that, for systems possessing homoclinic orbits, stipulates conditions by which to show they are chaotic. Therefore, one important step toward uncovering chaotic RG flow is to establish the existence of homoclinic RG flow.

In this short Letter, we study a QFT with global $O(N) \times$ $O(M)$ symmetry. Examining the RG flow of the theory as a function of $M$ and $N$, we determine the regime with nonmonotonic flow. In this regime, we establish the locations of a number of Bogdanov-Takens bifurcations, by which we conclude that the theory exhibits homoclinic RG flow. In other words, the model contains the fixed point with the peculiar property that a deformation by a relevant operator induces a flow that leads back to the original point: an RG flow where the IR and UV theories are one and the same. Homoclinic RG flow can be thought of as interpolating between the familiar type of RG flow (where a system flows from one fixed point to another) and the more exotic RG limit cycles (like limit cycles, homoclinic orbits are closed). In unitary QFTs, homoclinic RG flows are still forbidden by $c, a, F$ theorems, but a fixed point situated in a homoclinic orbit could possibly be described by a standard CFT, in contrast to fixed points undergoing a Hopf bifurcation, and which require operators with complex scaling dimensions.

The method we adopt can be applied more broadly to find homoclinic orbits in two-parametric families of theories. We expect the phenomenon to be present in many other QFTs.

The model.-We consider an $\mathcal{N}=1$ supersymmetric model of interacting scalar superfields $\Phi_{a b}^{i}$ that is invariant under the action of an $O(N) \times O(M)$ group in $d=3-\epsilon$ dimensions. The superfields are traceless-symmetric matrices with respect to the action of an $O(N)$ group and vectors under the action of an $O(M)$ group. There are four singlet marginal operators

$$
\begin{aligned}
& O_{1}=\operatorname{tr}\left[\Phi^{i} \Phi^{i} \Phi^{j} \Phi^{j}\right], \quad O_{2}=\operatorname{tr}\left[\Phi^{i} \Phi^{j} \Phi^{i} \Phi^{j}\right] \\
& O_{3}=\operatorname{tr}\left[\Phi^{i} \Phi^{i}\right]^{2}, \quad O_{4}=\operatorname{tr}\left[\Phi^{i} \Phi^{j}\right] \operatorname{tr}\left[\Phi^{i} \Phi^{j}\right]
\end{aligned}
$$

and so the full action is

$$
S=\int d^{d} x d^{2} \theta\left(\operatorname{tr}\left[\Phi^{i} D_{\alpha}^{2} \Phi^{i}\right]+\sum_{i} g_{i} O_{i}\right) .
$$

The RG flow of this model is gradient. We can find a function $F\left(g_{i}\right)$ and a four-by-four matrix $G_{i j}$ such that the beta functions are

$$
\beta_{i}=\mu \frac{d g_{i}}{d \mu}=G_{i j} \frac{\partial F}{\partial g_{j}} .
$$

If $G_{i j}$ is positive or negative definite, this equation implies that $F$ changes monotonically with the RG flow, so that cyclic and homoclinic flow lines are impossible. By explicit computation to leading order in perturbation theory, we find that the metric has determinant

$$
\begin{aligned}
\operatorname{det} G= & \frac{1}{4}(M-1)^{2}(M+2)^{2}(N-3) \\
& \times(N-2)^{2}(N+1)^{2}(N+4)^{2}(N+6) .
\end{aligned}
$$

We list the beta functions and the components of the metric in Supplemental Material Sec. B [36]. The zeros in $\operatorname{det} G$ occur because of linear relations among the four operator of the theory at special values of $M$ and $N$, and their presence indicates that eigenvalues change sign as $N$ and $M$ are varied. Indeed one can check that the metric is sign indefinite if $M \in(-2,1)$ or $N \in(-6,3)$, so that unusual RG flows are possible in this regime, and operators may develop complex scaling dimensions at "spooky" fixed points [24]. At integer values of $N$ and $M$, such operators are identically zero owing to the linear relations between the operators. The situation is closely analogous to the occurrence of evanescent operators at noninteger spacetime dimensions [37-42].

In the following, we will allow $M$ and $N$ to assume general real values. In consequence of this analytic extension, we observe Hopf bifurcations taking place in the model along various curves in the space of $M$ and $N$. But while Hopf bifurcations are a codimension-one bifurcation widely found in one-parameter systems of autonomous differential equations, we are dealing with a two-parameter system, and they exhibit a richer variety of flows. The possible codimension-two bifurcations can be classed into five types [2,3]-Bautin, Bogdanov-Takens, cusp, double-Hopf, and zero-Hopf-which signal different kinds of flow not present in generic one-parameter systems. As we shall now see, some of these possibilities are realized by the QFT with action (2).

Bogdanov-Takens bifurcation.-A Bogdanov-Takens bifurcation occurs generically when, at a fixed point, two eigenvalues of the stability matrix $\left(\partial \beta_{i} / \partial g_{j}\right)$ tend to zero as two bifurcation parameters $M$ and $N$ are appropriately tuned. The following equations must then be satisfied: 


$$
\begin{aligned}
& \beta_{i}\left(g_{i}, N, M\right)=0, \quad \operatorname{det}\left(\frac{\partial \beta_{i}}{\partial g_{j}}\right)\left(g_{i}, N, M\right)=0, \\
& \operatorname{tr}\left[\Lambda^{3}\left(\frac{\partial \beta_{i}}{\partial g_{j}}\right)\right] \equiv \operatorname{det}\left(\frac{\partial \beta_{i}}{\partial g_{j}}\right) \operatorname{tr}\left[\left(\frac{\partial \beta_{i}}{\partial g_{j}}\right)^{-1}\right]=0 .
\end{aligned}
$$

Written in the form (5), we see that the conditions for a Bogdanov-Takens (BT) bifurcation are polynomial equations in $g_{i}, M$, and $N$, and by Bézout's theorem there exist a finite number of points that satisfy these conditions. We refer to such points as BT points. For the QFT we are studying perturbatively, the beta functions exhibit several such points, as shown in Supplemental Material [36]. Their existence can be checked to high numerical accuracy with the use of standard programs [43]. Higher-loop contributions will provide corrections to the precise locations of these points, but as long as $\epsilon$ is sufficiently small, higherorder corrections will not alter the number or qualitative behavior of BT points.

While two eigenvalues tend to zero as we approach a BT point, right at the BT point itself we do not have a pair of eigenvectors with zero eigenvalues, because two respective eigenvectors usually become linearly dependent. Rather, the stability matrix at a BT point has a Jordan block of size two with zero eigenvalue (see Supplemental Material Sec. A [36]). This means that the theory at the BT point possesses two operators $\mathcal{O}_{1,2}$ such that the generator $D$ of dilatations acts in the following way:

$$
D \mathcal{O}_{1}=d \mathcal{O}_{1}, \quad D \mathcal{O}_{2}=d \mathcal{O}_{2}+\mathcal{O}_{1}
$$

The possibility of indecomposable representations of the conformal group was extensively studied in $[44,45]$. The upshot is that the BT theory constitutes a logarithmic CFT containing generalized marginal operators $\mathcal{O}_{1,2}$. In consequence, BT theories are nonunitary and we have

$$
\left\langle\mathcal{O}_{2}(0) \mathcal{O}_{2}(x)\right\rangle=-\frac{2 k_{\mathcal{O}} \log |x|}{|x|^{2 d}}, \quad\left\langle\mathcal{O}_{1}(0) \mathcal{O}_{2}(x)\right\rangle=\frac{k_{\mathcal{O}}}{|x|^{2 d}},
$$

for some constant $k_{\mathcal{O}}$.

The conditions (5) are not entirely sufficient for a BT bifurcation. One must also require smoothness and a set of inequalities that are generically true. Violations of the inequalities typically require fine-tuning of additional parameters. Incidentally, at the integer values $M=2$ and $N=3$, right on the boundary of the regimes with monotonic and nonmonotonic RG flows, we observe a fixed point that satisfies (5), but which fails to meet these genericity requirements and hence is not described by a logarithmic CFT.

In Supplemental Material Sec. A [36] we give the precise statement of the Bogdanov-Takens bifurcation theorem, and we explicitly check that it applies to a BT point in the QFT we are studying, situated at $M \approx 0.2945$ and $N \approx 4.036$. What this means is that we can transform the beta functions near the BT point into a particularly simple form, known as Bogdanov normal form:

$$
\begin{aligned}
& \dot{\eta}_{1}=\eta_{2}, \\
& \dot{\eta}_{2}=\delta_{1}+\delta_{2} \eta_{1}+\eta_{1}^{2}+s \eta_{1} \eta_{2}+\mathcal{O}\left(|\eta|^{3}\right), \\
& \dot{\eta}_{i}=\lambda_{i} \eta_{i} \quad \text { for } i>2,
\end{aligned}
$$

where $s=-1$, and $\delta_{1,2}$ are functions of $N$ and $M$ that vanish right at the $\mathrm{BT}$ point.

By bringing the system into normal form, we can use Eqs. (7) to determine the behavior of the system for small enough $\delta_{1}$ and $\delta_{2}$. In particular, we can constrain ourselves to studying the surface where only $\eta_{1}$ and $\eta_{2}$ are nonzero, noting that the dynamics in the transverse directions $\eta_{3}$ and $\eta_{4}$ are quite simple. Depending on $\delta_{1}$ and $\delta_{2}$, the flow of $\eta_{1,2}$ falls into different topological types. The classification can be found in textbook [46] and amounts to the following. In the vicinity of the BT point at $\delta_{1}=\delta_{2}=0$, there are four regimes with qualitatively different flows:

Regime (1: The flow has no fixed point.

In the other three regimes, the flow has two fixed points, which we will label left and right. The right point is always a saddle point.

Regime (2): The left point is unstable, and all flow lines starting near it terminate at the right fixed point.

Regime (3): The left point is now stable, and a repulsive limit cycle separates the two fixed points.

Regime (4): The left point is still stable, but the limit cycle has disappeared. Some flow lines starting near the right fixed point terminate at the left fixed point. Regions (3) and (4) are separated by a saddle homoclinic bifurcation along $\delta_{1}=-(6 / 25) \delta_{2}^{2}+\cdots, \delta_{2}<0$. In the case of the BT point at $(M, N) \approx(0.2945,4.036)$, the locations of these four adjoining regimes, as computed in Supplemental Material Sec. A [36], are shown in Supplemental Material Fig. 1. And the RG flow in each regime is depicted in Fig. 1.

A saddle-node bifurcation corresponds to the collision and disappearance of two equilibria in dynamical systems. The phenomenon has already been observed in the critical $O(N)$ model [47], prismatic models [48], and in $\mathrm{QCD}_{4}$ [49-52].

An Andronov-Hopf bifurcation represents a change of stability at a fixed point that has complex eigenvalues. The flow near the fixed point gives birth to a limit cycle. This RG flow bifurcation was recently studied in [24].

The most interesting and new phenomenon associated with the model of the present Letter happens along the homoclinic bifurcation line. Here the flow exhibits what is known as a homoclinic orbit.

Homoclinic $R G$ flow.-A homoclinic orbit is a flow line that connects a stable and an unstable direction of a saddle point. Figure 2 depicts the kind of homoclinic orbit generated by a BT bifurcation, with the saddle point marked by a red dot. The homoclinic orbit envelops another 


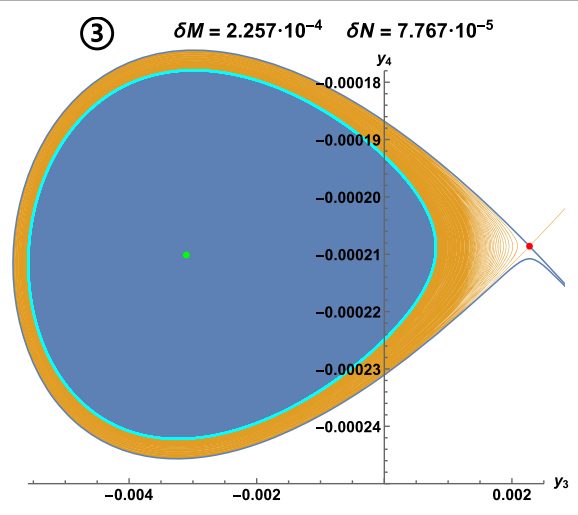

RG flow in region (3). An IR-repulsive limit cycle, marked in cyan, separates the two fixed points.

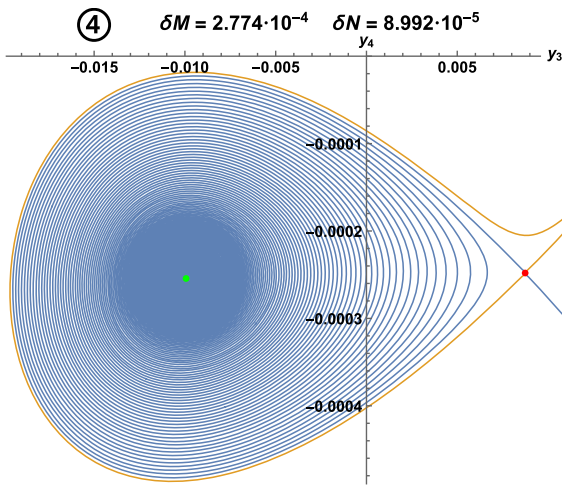

RG flow in region (4). In passing from region (3) to (4) the limit cycle collided with the red fixed point in a homoclinic bifurcation.

FIG. 1. The topologically distinct types of RG flow in the vicinity of the Bogdanov-Takens bifurcation at $M=$ $M^{*} \approx 0.2945$ and $N=N^{*} \approx 4.036$. The variables $y_{3}$ and $y_{4}$ are linear combinations of the four coupling constants $g_{i}$, with precise definitions given in Supplemental Material Sec. A [36], and $\delta M=M-M^{*}, \delta N=N-N^{*}$.

fixed point marked in green. In a QFT context, the green point is "spooky": the couplings are real, but the stability matrix $\left(\partial \beta_{i} / \partial g_{j}\right)$ has complex eigenvalues. In contrast to such spooky points, and to complex CFTs [51,53], the red saddle point is associated with real couplings and real eigenvalues of the stability matrix. These eigenvalues are small and have opposite signs: $\lambda_{1},-\lambda_{2} \ll 1$. The positive eigenvalue corresponds to a slightly relevant operator $\mathcal{O}_{1}$ with dimension $\Delta_{1}=d+\lambda_{1}>d$, and the negative eigenvalue to a slightly irrelevant operator $\mathcal{O}_{2}$ with dimension $\Delta_{2}=d+\lambda_{2}<d$. In this sense, the red saddle point corresponds to a real CFT.

Standard RG lore states that if we perturb a system in the direction of a relevant operator, then we expect for the system to lose conformality altogether or to flow to a

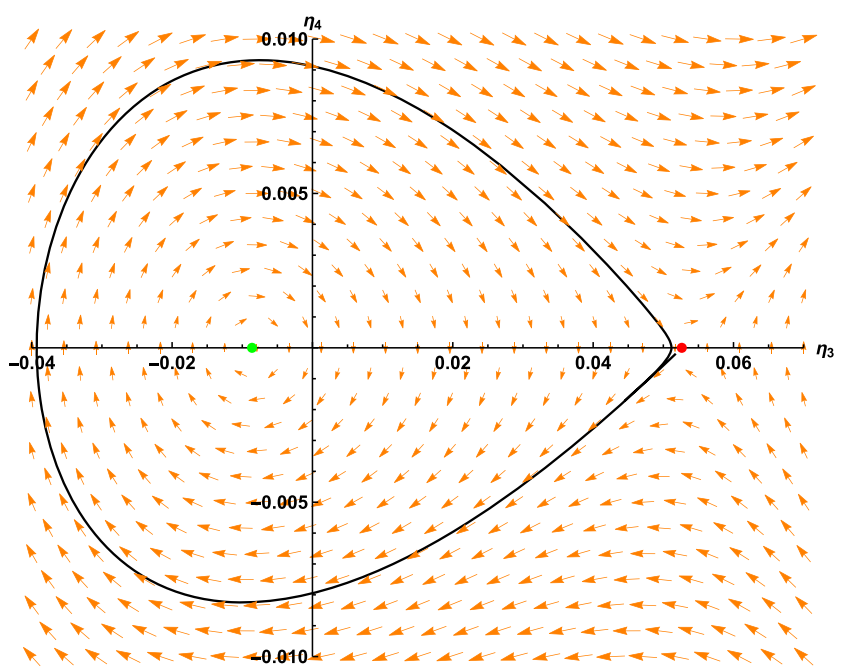

FIG. 2. Flow diagram for a dynamical system containing a homoclinic orbit (marked in black), i.e., a flow line that starts and ends at the same point. The system is described by Eqs. (7) with parameters $\delta_{1}=-0.000453178$ and $\delta_{2}=-0.0440214$. The red and green dots indicate fixed points. The green dot is a "spooky" fixed point. The theory at the red dot is a homoclinic CFT.

different CFT. In the terminology of dynamical systems, standard RG trajectories are heteroclinic orbits. The classical example is the Wilson-Fisher fixed point: by perturbing a Gaussian theory in $4-\epsilon$ dimension we flow to a weakly coupled interacting CFT, which in three dimensions interpolates to the Ising model. Homoclinic bifurcations provide exotic counterexamples to this general picture: if we perturb the system in the direction of a relevant operator, we come back to the original fixed point, which we can term a homoclinic CFT. Such RG behavior obviously violates the $F$ theorem so that homoclinic fixed points must be nonunitary, as is generally the case for CFTs with symmetry groups of noninteger rank [26].

If we tune the bifurcation parameters to approach the BT point along the saddle homoclinic bifurcation (the red curve in Supplemental Material Fig. 1 [36]), then the homoclinic orbit shrinks to a point and vanishes. In this limit, the red homoclinic CFT and the green spooky fixed point merge and become a logarithmic CFT.

Zero-Hopf bifurcations: The road to chaos.-The Bogdanov-Takens bifurcation is not the only codimension-two bifurcation that can be observed to take place in the model (2). The theory also possesses two points in the space of $g_{i}, M$, and $N$ where the stability matrix has a pair of purely imaginary eigenvalues and one zero eigenvalue. Such fixed points indicate what is known as a Zero-Hopf (ZH) or a Fold-Hopf bifurcation. This type of bifurcation was classified in [54] and can be divided into six subtypes. In the notation of [2], the model has a type I ZH bifurcation at $(M, N) \approx(0.8447,-1.807)$ and a type IIa ZH bifurcation at $(M, N) \approx(-3.816,1.188)$. At a type I bifurcation point, a saddle-node bifurcation is incident to a pitchfork 
bifurcation, and there are no nearby cyclic orbits. At a type IIa point, a saddle-node bifurcation is again incident to a pitchfork bifurcation, but additionally a Hopf bifurcation is also incident to the point, except that the stability coefficient of the associated limit cycle (what was referred to as the Hopf constant in [24]) exactly vanishes in a quadratic approximation, so that cubic fluctuations or higher decide the fate of the cyclic flow near a type IIa point.

Generally, ZH bifurcation points are of particular interest because it is known that in their vicinity what is known as a Shilnikov homoclinic orbit may develop and render the system chaotic [35,46]. Recently it was proven in [55] that the presence of $\mathrm{ZH}$ bifurcations of type III guarantees the existence of a Shilnikov orbit and a nearby infinite set of saddle periodic orbits. This nontrivial invariant set can be embedded in an attracting domain, thus implying Shilnikov chaos.

The $\mathrm{ZH}$ points of the model in the present Letter are not of type III, and we cannot claim that the system is chaotic. It may be worthwhile to investigate if there exist other models that meet the simple criteria for the assured appearance of chaos.

Conclusion and outlook.-The approach suggested and adopted in $[4,24,52]$ of studying the beta functions and renormalization of QFTs from the general perspective of dynamical systems provides a method of understanding the full range of possible RG flows. A powerful tool to this end is offered by Bogdanov's and Taken's bifurcation theorem [27], which lists a simple set of conditions that guarantee the existence of a homoclinc RG orbit, and which can be checked already at first order in perturbation theory.

In this short Letter we have presented a QFT that satisfies these conditions, namely a supersymmetric model with global symmetry group $O(N) \times O(M)$, where $N$ and $M$ play the role of the bifurcation parameters of the system. We determined a number of parameter values where a BT bifurcation takes place and investigated the nearby RG flow to uncover the presence of homoclinic orbits, where the perturbation of a fixed point by a relevant operator induces an RG flow that returns to its starting point along an irrelevant direction. This kind of flow does not violate the $F$ theorem since it occurs in a nonunitary regime of the theory: at noninteger values of $d, M$, and $N$. Homoclinic RG flow may also be possible in a unitary theory with a multivalued $F$ function.

Nonunitary QFTs have a number of physical applications, such as percolation and random walks [56], though the considered model (2) with fractional $d, M$, and $N$ would be very hard to find in nature or realize in an experimental setup. However, we could work directly in $d=3$ dimensions by introducing a gauge field and turning on a Chern-Simons term, which would generate terms in the beta functions that are linear in the couplings. And theories with symmetry groups of fractional rank can be realized in lattice models, for example the $O(N)$ loop models introduced in [57]. In this setting, homoclinic RG flow would provide an interesting form of UV completion where a model is described by the same CFT in the UV and the IR. Moreover, it would present a rare example of the phenomenon of self-tuning in the presence of an unstable direction: one could alter the corresponding lattice coupling and still flow to the same fixed point. For the present model, however, tuning would still be required since we have two unstable directions and the self-tuning only applies to one of these.

There are several bifurcation theorems that give simple criteria for other novel kinds of RG flows $[2,3,46]$. Some of these theorems allow for the determination of the onset of chaotic flow based on straightforward computations around fixed points [55]. It would be interesting to find out if QFTs give birth to chaos when $N$ becomes fractional.

We are grateful to Igor R. Klebanov for very insightful discussions and suggestions throughout the project. We are also grateful to Alexander Gorsky, Alexei Milekhin, Yuri Kuznetsov, Alexander Polyakov, Sergey Gukov, Slava Rychkov, and Bernardo Zan for valuable discussions and comments. We also thank Maikel Bosschaert for spotting a considerable typo in Supplemental Material Sec. B [36]. This research was supported in part by the U.S. NSF under Grant No. PHY-1914860.

[1] K. G. Wilson and J. B. Kogut, The renormalization group and the epsilon expansion, Phys. Rep. 12, 75 (1974).

[2] J. Guckenheimer and P. Holmes, Nonlinear Oscillations, Dynamical Systems, and Bifurcations of Vector Fields (Springer Science \& Business Media, New York, 2013), Vol. 42.

[3] V. I. Arnold, Geometrical Methods in the Theory of Ordinary Differential Equations (Springer Science \& Business Media, New York, 2012), Vol. 250.

[4] S. Gukov, RG flows and bifurcations, Nucl. Phys. B919, 583 (2017).

[5] M. J. Feigenbaum, Quantitative universality for a class of nonlinear transformations, J. Stat. Phys. 19, 25 (1978).

[6] A. B. Zamolodchikov, Irreversibility of the flux of the renormalization group in a $2 \mathrm{D}$ field theory, JETP Lett. 43, 730 (1986).

[7] Z. Komargodski and A. Schwimmer, On renormalization group flows in four dimensions, J. High Energy Phys. 12 (2011) 099.

[8] M. A. Luty, J. Polchinski, and R. Rattazzi, The $a$-theorem and the asymptotics of 4D quantum field theory, J. High Energy Phys. 01 (2013) 152.

[9] I. R. Klebanov, S. S. Pufu, and B. R. Safdi, F-theorem without supersymmetry, J. High Energy Phys. 10 (2011) 038.

[10] D. L. Jafferis, I. R. Klebanov, S. S. Pufu, and B. R. Safdi, Towards the $F$-theorem: $N=2$ field theories on the three-sphere, J. High Energy Phys. 06 (2011) 102. 
[11] H. Casini and M. Huerta, On the RG running of the entanglement entropy of a circle, Phys. Rev. D 85, 125016 (2012).

[12] A. Morozov and A. J. Niemi, Can renormalization group flow end in a big mess? Nucl. Phys. B666, 311 (2003).

[13] T. L. Curtright, X. Jin, and C. K. Zachos, RG Flows, Cycles, and $c$-Theorem Folklore, Phys. Rev. Lett. 108, 131601 (2012).

[14] D. Bernard and A. LeClair, Strong weak coupling duality in anisotropic current interactions, Phys. Lett. B 512, 78 (2001).

[15] A. LeClair, J. M. Roman, and G. Sierra, Log periodic behavior of finite size effects in field theories with RG limit cycles, Nucl. Phys. B700, 407 (2004).

[16] A. Leclair, J.M. Roman, and G. Sierra, Russian doll renormalization group, Kosterlitz-Thouless flows, and the cyclic sine-Gordon model, Nucl. Phys. B675, 584 (2003).

[17] S. D. Glazek and K. G. Wilson, Renormalization of overlapping transverse divergences in a model light front Hamiltonian, Phys. Rev. D 47, 4657 (1993).

[18] S. D. Glazek and K. G. Wilson, Limit Cycles in Quantum Theories, Phys. Rev. Lett. 89, 230401 (2002); 92, 139901 (E) (2004).

[19] K. M. Bulycheva and A. S. Gorsky, Limit cycles in renormalization group dynamics, Phys. Usp. 57, 171 (2014).

[20] A. Gorsky and F. Popov, Atomic collapse in graphene and cyclic renormalization group flow, Phys. Rev. D 89, 061702 (R) (2014).

[21] A. LeClair, J. M. Roman, and G. Sierra, Russian doll renormalization group and superconductivity, Phys. Rev. B 69, 020505(R) (2004).

[22] E. Braaten and D. Phillips, The renormalization group limit cycle for the $1 / r^{2}$ potential, Phys. Rev. A 70, 052111 (2004).

[23] S. M. Dawid, R. Gonsior, J. Kwapisz, K. Serafin, M. Tobolski, and S. D. Głazek, Renormalization group procedure for potential $-g / r^{2}$, Phys. Lett. B 777, 260 (2018).

[24] C. B. Jepsen, I. R. Klebanov, and F. K. Popov, RG limit cycles and unconventional fixed points in perturbative QFT, Phys. Rev. D 103, 046015 (2021).

[25] P.-G. De Gennes and P.-G. Gennes, Scaling Concepts in Polymer Physics (Cornell University Press, Ithaca, 1979).

[26] D. J. Binder and S. Rychkov, Deligne categories in lattice models and quantum field theory, or making sense of $O(N)$ symmetry with non-integer $N$, J. High Energy Phys. 04 (2020) 117.

[27] E. Hopf, Bifurcation of a periodic solution from a stationary solution of a system of differential equations, Berlin Math. Phys. Klasse, Sachsischen Akad. Wiss. Leipzig 94, 3 (1942).

[28] R. Bogdanov, Bifurcations of a limit cycle for a family of vector fields on the plane, Sel. Math. Sov. 1, 373 (1981).

[29] F. Takens, Forced oscillations and bifurcations, Appl. Global Anal. I 3, 1 (2001).

[30] P. Cvitanovic, Universality in Chaos (CRC Press, Boca Raton, 2017), ISBN 9780852742600.

[31] D. Ruelle and F. Takens, On the nature of turbulence, Les Rencontres Phys. Math. Strasbourg-RCP25 12, 1 (1971).
[32] V. E. Zakharov, V. S. L'vov, and G. Falkovich, Kolmogorov Spectra of Turbulence I: Wave Turbulence (Springer Science \& Business Media, New York, 2012).

[33] E. N. Lorenz, Deterministic nonperiodic flow, J. Atmos. Sci. 20, 130 (1963).

[34] D. J. Gross and V. Rosenhaus, Chaotic scattering of highly excited strings, J. High Energy Phys. 05 (2021) 048.

[35] L. P. Shilnikov, A case of the existence of a denumerable set of periodic motions, Dokl. Akad. Nauk SSSR 160, 558 (1965),

[36] See Supplemental Material at http://link.aps.org/ supplemental/10.1103/PhysRevLett.127.141602 for the expressions for two-loop beta-functions for the theory considered in the paper, bifurcation conditions for these equations and transformation of these equations to the normal form.

[37] J. C. Collins, Renormalization: An Introduction to Renormalization, The Renormalization Group, and the Operator Product Expansion, Cambridge Monographs on Mathematical Physics Vol. 26 (Cambridge University Press, Cambridge, 1986).

[38] M. Bos, An example of dimensional regularization with antisymmetric tensors, Ann. Phys. (N.Y.) 181, 177 (1988).

[39] M. J. Dugan and B. Grinstein, On the vanishing of evanescent operators, Phys. Lett. B 256, 239 (1991).

[40] J. A. Gracey, Four loop MS-bar mass anomalous dimension in the Gross-Neveu model, Nucl. Phys. B802, 330 (2008).

[41] M. Hogervorst, S. Rychkov, and B. C. van Rees, Truncated conformal space approach in $d$ dimensions: A cheap alternative to lattice field theory? Phys. Rev. D 91, 025005 (2015).

[42] M. Hogervorst, S. Rychkov, and B. C. van Rees, Unitarity violation at the Wilson-Fisher fixed point in $4-\epsilon$ dimensions, Phys. Rev. D 93, 125025 (2016).

[43] Pydstool homepage, https://pydstool.github.io/PyDSTool/ ProjectOverview.html.

[44] V. Gurarie, Logarithmic operators in conformal field theory, Nucl. Phys. B410, 535 (1993).

[45] M. Hogervorst, M. Paulos, and A. Vichi, The ABC (in any D) of logarithmic CFT, J. High Energy Phys. 10 (2017) 201.

[46] Y. A. Kuznetsov, Elements of Applied Bifurcation Theory (Springer Science \& Business Media, New York, 2013), Vol. 112.

[47] L. Fei, S. Giombi, I. R. Klebanov, and G. Tarnopolsky, Three loop analysis of the critical $O(N)$ models in $6-\varepsilon$ dimensions, Phys. Rev. D 91, 045011 (2015).

[48] S. Giombi, I. R. Klebanov, F. Popov, S. Prakash, and G. Tarnopolsky, Prismatic large $N$ models for bosonic tensors, Phys. Rev. D 98, 105005 (2018).

[49] H. Gies and J. Jaeckel, Chiral phase structure of QCD with many flavors, Eur. Phys. J. C 46, 433 (2006).

[50] D. B. Kaplan, J.-W. Lee, D. T. Son, and M. A. Stephanov, Conformality lost, Phys. Rev. D 80, 125005 (2009).

[51] V. Gorbenko, S. Rychkov, and B. Zan, Walking, weak firstorder transitions, and complex CFTs, J. High Energy Phys. 10 (2018) 108.

[52] F. Kuipers, U. Gürsoy, and Y. Kuznetsov, Bifurcations in the RG-flow of QCD, J. High Energy Phys. 07 (2019) 075. 
[53] V. Gorbenko, S. Rychkov, and B. Zan, Walking, weak firstorder transitions, and complex CFTs II. Two-dimensional Potts model at $Q>4$, SciPost Phys. 5, 050 (2018).

[54] F. Takens, Singularities of vector fields, Publ. Math. Inst. Hautes Études Sci. 43, 47 (1974).

[55] I. Baldomá, S. Ibánez, and T. M. Seara, Hopf-zero singularities truly unfold chaos, Commun. Nonlinear Sci. Numer. Simul. 84, 105162 (2020).
[56] J. Cardy, Logarithmic conformal field theories as limits of ordinary CFTs and some physical applications, J. Phys. A 46, 494001 (2013).

[57] E. Domany, D. Mukamel, B. Nienhuis, and A. Schwimmer, Duality relations and equivalences for models with $\mathrm{O}(N)$ and cubic symmetry, Nucl. Phys. B190, 279 (1981). 